\title{
Hardware-in-the-Loop Validation of Control Parameters for MVDC Shipboard Power Systems
}

\author{
Edoardo De Din, Gianluca Lipari, Marco Cupelli, Antonello Monti \\ Institute for Automation of Complex Power Systems \\ E.ON Energy Research Center RWTH Aachen University \\ Aachen, Germany \\ [ededin, glipari, mcupelli, amonti]@eonerc.rwth-aachen.de
}

\begin{abstract}
Power-electronics-based Medium Voltage Direct Current (MVDC) shipboard power systems, consisting of several interconnected feedback-controlled switching converters, suffer from potential degradation of stability induced by negative incremental resistances due to the presence of constant power loads (CPLs). Several bus stabilizing control approaches have been presented in the past. In this work a novel Control Parameter tuning procedure, applicable to various control approaches, is introduced and implemented in LabView. The main features of the Control parameter tuning include the implementation of the Particle Swarm Optimization (PSO) method and the interface with the FPGAs, where the control methods are implemented. The on-line optimization procedure allows the fair comparison of the different control algorithms. Test are carried out for a shipboard power system based on the IEEE 1709 standard, using the Hardware-in-the-Loop (HIL) simulation setup together with the Real Time Digital Simulator (RTDS). The test shows the feasibility and effectiveness of the PSO to perform the on-line tuning of different controllers and enable a fair comparison between them.
\end{abstract}

Keywords-Labview, Power System Stability, Optimization, Hardware-in-the-Loop, Control Systems, Nonlinear Systems

\section{INTRODUCTION}

According to the U.S. Navy, addressing the key technical issue of bus voltage regulation will enable the deployment of Medium-Voltage Direct Current (MVDC) shipboard power systems [1] [2]. In MVDC shipboard power systems, the individual loads, or groups of loads, are normally fed through power electronic converters directly connected to the distribution MVDC bus. The load converters, under tight feedback regulation, behave at their input terminals as Constant Power Loads (CPLs) within their control loop bandwidth. In the small-signal sense, these CPLs exhibit a negative incremental input resistance behavior, which may be the origin of the undesired destabilizing effect [3]-[4]. Conditions under which the instability takes place and methods for the stability analysis of MVDC systems have been reviewed and discussed in [5].

Recently, the MVDC Integrated Ship Power Systems (ISPSs) efforts have been focused on the design of load converters, i.e. those interfacing the MVDC distribution bus and the loads. In order to prevent the MVDC ISPS from voltage instability, the small-signal negative input resistance behavior of the load converters can be relaxed either through dedicated control algorithms [6], through the introduction of stabilizing virtual impedances [7], or through forcing disconnection before the MVDC system operates out of the stable range [7]. The control algorithms can reveal some troubles on tuning their parameters, because of the lacking knowledge of the system or due to the difficulties to correlate the parameters of the controllers and their effects on the control performance. Moreover, moving from a numerical simulation to an experimental setup, introduces some uncertainties, such as delays or noises, which complicate the tuning. In order to answer to these problems, an on-line optimization of the control parameters has been performed. The Particle Swarm Optimization (PSO) [8] method has been selected, operating in a Hardware-in-the-Loop simulation setup, where the control methods are implemented in the FPGAs and the MVDC Shipboard Power System is simulated in the Real Time Digital Simulator (RTDS). Another benefit that can be achieved from the application of an optimization technique on these control methods is the possibility of comparing them, by means of the evolution of the fitness function.

\section{Control Algorithms}

This section reviews the state of the art of the control algorithms, which can be used for bus voltage stabilization. The purpose of this analysis is to highlight key design and implementation aspects, and to provide a comprehensive bibliography. The model of the MVDC ISPS taken into consideration in this paper is based on [6], where the synchronous generators are connected to the main MVDC bus through a rectifier and a DC-DC converter. Replicating a feature of terrestrial power systems on ISPSs, the control algorithms are applied on the generation side, no matter which load is connected or disconnected, enabling the Plug\&Play capability for every load, and the usage of common off-the-shelf components.

The generation side control methods described in this paper can be categorized in centralized and decentralized. The centralized controllers are: the LSF (Section II.A), which is based on an exact input-output linearization of the plant and the centralized synergetic control (Section II.B), which uses a manifold to control the system. The decentralized approaches rely on the virtual disturbance concept (Section II.C), which models the non-local part of the power system as disturbance current sink, which models the non-local part as a power sink

\section{A. Linearizing State Feedback (LSF)}

By considering the nonlinear set of equations of the MVDC ISPS [9], an approach to properly control the bus voltage is offered by the LSF technique [9][6]. This is a control technique which is based on exact input-output linearization. It is applied in centralized control architecture, assuming therefore that the LSF controller is able to collect all the measurements related to each converter and load (i.e. voltage and current). This actually means that all the relevant states to be controlled are known and available. The overall schematic of this control approach is depicted in Figure 1. After the exact-input output linearization, which compensates the nonlinearity, a linear system is obtained where classical pole placement technique can be used so that the natural frequency and damping coefficient are shifted to the desired values [9]. Instead of using a single primary voltage regulator 
as described in [9], here the voltage regulation is realized with a PI controller for each generation side converter.

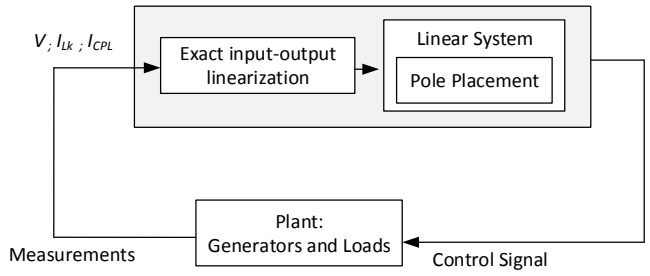

Figure 1. Schematic of LSF controller

\section{B. Centralized Synergetic Control}

In contrast to linear control, synergetic control is capable of handling system nonlinear dynamics and ensuring global system stability [6], while systems consisting of parallel connected power converters under linear control may not show proper behavior and may lead to system collapse [10]. This control technique uses constant switching frequency, which makes it appealing to implement in power converters. This control law uses a model of the system and forces the system onto a manifold. After the synergetic control design is completed an analytical control law is the result which ensures stable motion to the equilibrium point of the closed loop system [12]. The manifold design now includes all LRCs. The overall procedure depicted in Figure 2 was laid out in [12]-[13].

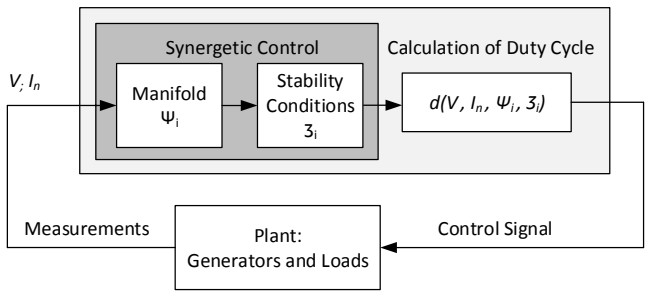

Figure 2 Synergetic Control

\section{Virtual Disturbance based controllers}

In this subsection, a concept of decentralized control architecture is presented. It assumes, by estimation process, a linearized system on which linear and nonlinear control laws can be applied [11], [12], [14], [15]. The estimation process is performed by a Kalman filter which uses stochastic framework. The idea is to lump all nonlocal source converters and loads in a virtual disturbance current sink $I_{d}$ depicted in Figure 3. A local control acts on two states, the bus voltage $V$ and the inductor current $I_{L}$, whereas the virtual disturbance is assumed to have unknown dynamics. The Kalman filter, which is augmented by an additional state $I_{d}$, can be used to observe the current $I_{L}$ and estimate $I_{d}$ which results in a total of three states [12].

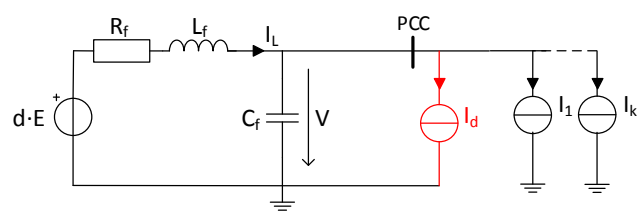

Figure 3. Decentralized control design model

The control strategies are based on the averaged model of a buck converter in Continuous Conduction Mode (CCM), where $V$ is the voltage across the capacitor and and $I_{L}$ the inductor current. $E$ is the input voltage of the buck converter, $d$ the duty cycle and $L_{f}, R_{f}$ and $C_{f}$ the converter output filter values. It has to be highlighted that the disturbance current $I_{d}$ is included in the system description and it is assumed to be a known quantity at this stage of the design process. All the following controllers are designed according to the separation principle, which allows the design of the observer and controller independently from each other. As a consequence, during the control law design phase, the current $I_{d}$ is seen as a deterministic quantity. Figure 4 depicts an overview of the general architecture of virtual disturbance based controllers. The control law can be linear as the LQG controller [12] or nonlinear as the Backstepping [14] and the Synergetic Control [12].

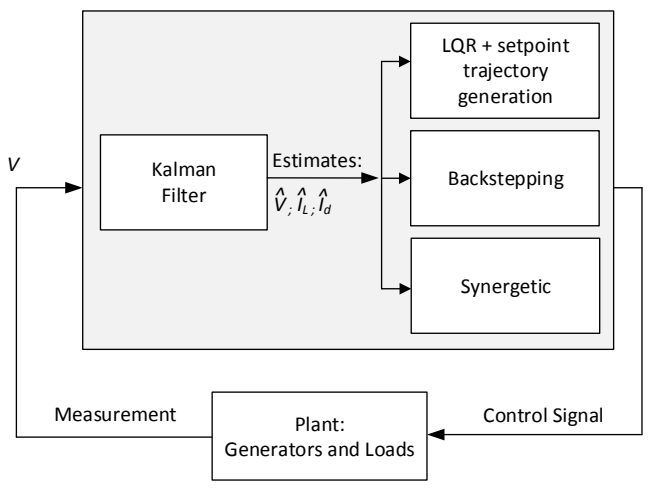

Figure 4. Overview Decentralized Controllers

\section{EXPERIMENTAL SETUP}

The experimental setup consists of three different parts, as can be observed in Figure 5. The RT host PC, governed by the LABVIEW RT operating system, which executes the PSO algorithm, four NIPCIe7841R devices, containing the Xilinx Virtex5 LX30, an FPGA board responsible of executing the control algorithms, and the Real Time Digital Simulator (RTDS) from RTDS Technologies, which performs the simulation of the electrical components of the shipboard power system.

The RT host obtains the voltage measurement, necessary for the evaluation of the cost function, directly from the ADC converter of one of the FPGA devices. For every particle generated, the RT host runs the control algorithm and sends the parameters of the control strategy to the FPGAs, based on the values of the particle. The simulation operates for 20 seconds, where the integral of the absolute voltage error is calculated with a time step of $2 \mathrm{~ms}$. The signals among the RT host and the FPGA are exchanged through the Ethernet connection. During the simulation time of a single particle, a load step is performed in RTDS, in order to take into account the effect of the load step in the optimization process. 


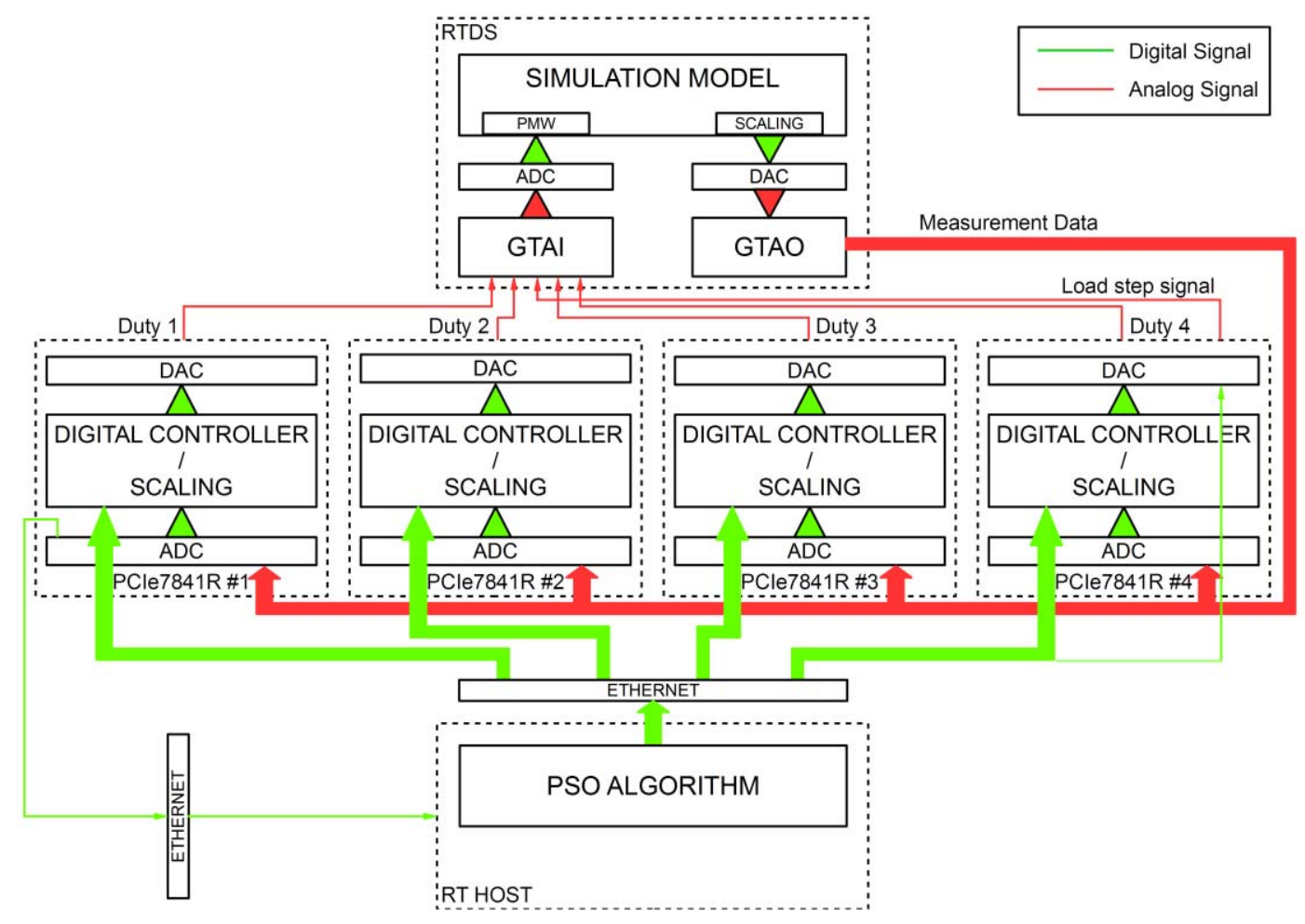

Figure 5. The experimental setup

This is realized with a signal sent by the RT host to the RTDS analog input interface board (GTAI) using the DAC of one of the PCIe devices, as described in Figure 5. This signal controls the switch that varies the load level of the ship power system simulated in RTDS.

The four PCIe7841R devices perform the control strategies, based on the parameters coming from PSO algorithm, depicted in green, and the analog measurements, depicted in red, of the simulated system, converted into digital signals. The 16-bit integer measurements are then adapted to the fixed-point execution on the FPGA and, for the virtual based control strategies, a pre-tuning of the Kalman Filter Parameters is performed to compensate for the undesired background noise due to quantization and cabling. The parallel operation of the four FPGAs is obtained with a signal generator producing a square waveform of $50 \mathrm{kHz}$, which synchronizes the execution of the control algorithms in the FPGAs with $20 \mu$ s loop time. The droop strategy for governing the current sharing was used for all stabilizing control methods and implemented in the FPGA calculation. The values of the duty cycles are then scaled and converted into analog signals and sent to the GTAI board, which realizes the digital conversion.

The simulation of the ship power system under test is executed in RTDS and is characterized by four generation lines, each one composed by a synchronous generator which feeds a buck converter by means of a 6-pulse diode bridge rectifier. Each buck converter detects a constant voltage source during the transient time, because of the slower dynamic of the synchronous machine compared to the power converter. The parameters of the RLC filter of the buck converters are defined in Table I. The load side is distinguished by two buck converters, which step down the voltage of the main bus to 3 $\mathrm{kV}$ and feed a variable load. They are controlled with a switching frequency of $1 \mathrm{kHz}$ and $3 \mathrm{kHz}$ respectively and governed by PID controllers. Besides these two, the load side is characterized by resistive linear load that represent a total amount of $2.8 \mathrm{MW}$, which is kept constant during the simulation. To avoid the steady state error after the load increase, a centralized secondary control loop consisting of a PI controller with the feedback of the bus voltage has been implemented in the RTDS framework [16], [18], [19]. The PWM modulation has been also implemented in RTDS in order to leave only the calculation of the control strategy inside the FPGA. The measurements obtained with the simulation are scaled and converted into analog signals and sent through the analog output interface board (GTAO) to the FPGAs.

Table I: Values of ISPS

\begin{tabular}{|l|c|c|c|}
\hline & $\begin{array}{c}\text { Source } \\
\mathbf{1 , 2 , 3 , 4} \\
(\text { Buck) }\end{array}$ & $\begin{array}{c}\text { POL } \\
\text { (Buck } \\
\mathbf{1 k H z}\end{array}$ & $\begin{array}{c}\text { POL } \\
\text { (Buck } \\
\text { 3kHz) }\end{array}$ \\
\hline $\mathbf{P}_{\mathbf{n}}[\mathbf{M W}]$ & 20 & 25 & 25 \\
\hline $\mathbf{V}_{\text {in }}[\mathbf{k V}]$ & 8.91 & 6 & 6 \\
\hline $\mathbf{V}_{\text {out }}[\mathbf{k V}]$ & 6 & 3 & 3 \\
\hline $\mathbf{f}_{\mathbf{s}}[\mathbf{k H z}]$ & 1 & 1 & 3 \\
\hline $\mathbf{R}_{\mathbf{f}}[\mathbf{m} \boldsymbol{\Omega}]$ & 99.7 & 33.2 & 33.2 \\
\hline $\mathbf{L}_{\mathbf{f}}[\mathbf{m H}]$ & 2.1 & 1.1 & 0.35 \\
\hline $\mathbf{C}_{\mathbf{f}}[\boldsymbol{\mu F}]$ & 659.72 & 2000 & 1100 \\
\hline $\mathbf{C}_{\text {if }}[\boldsymbol{\mu F}]$ & - & 3608 & 1327 \\
\hline $\mathbf{B a s e}_{\mathbf{m}} \mathbf{L o a d}[\mathbf{M W}]$ & 2.8 & - & - \\
\hline $\begin{array}{l}\mathbf{L o a d} \mathbf{I n c r e a s e} \\
{[\mathbf{M W}]}\end{array}$ & - & 11.5 & 11.5 \\
\hline
\end{tabular}

\section{PSO ALGORITHM}

The Particle Swarm Optimization method is a computational technique that makes use of a vector of particles moving in the space of the problem with a certain velocity. A review of the different applications of the PSO in power system can be found in [8].

The implementation of the PSO in the RT host, which has been realized in the LabVIEW framework, performs an on-line 
optimization of the parameters of the controllers. This configuration takes into account the effect of delays, noises and errors that are typical of Hardware in the Loop implementation that reveals to be difficult to evaluate and then to implement into off-line numerical simulations. Moreover, this configuration avoids the possibility of generating parameters for the controllers which show a very good behavior in the numerical simulation, without displaying the same performance in the experimental simulation, because of the noises that affect the signals and the slight errors on the estimation.

The PSO starts with the calculation of the first population of particles with random position, based on the following formula (1):

$$
\vec{x}_{\text {init }}=\left(\vec{x}_{\text {max }}-\vec{x}_{\text {min }}\right) \cdot \text { rand }+\vec{x}_{\text {min }}
$$

where $\vec{x}_{\text {max }}$ and $\vec{x}_{\text {min }}$ are the boundaries of the hyperspace problem.

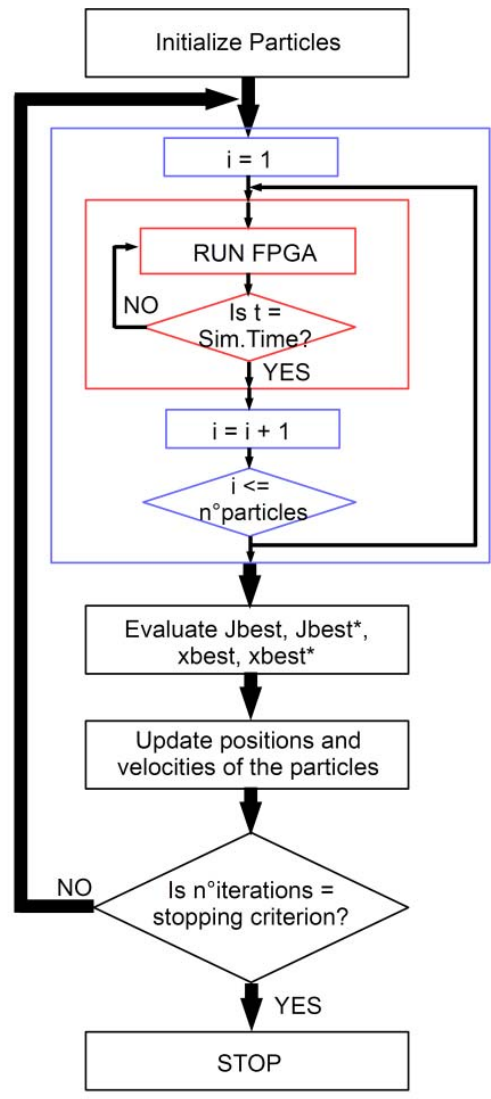

Figure 6. The PSO Algorithm implemented in the RT host

Then, the vector of particles enters in a for cycle, depicted in blue in Figure 6, which decomposes the vector. Then, for each single particle, the FPGAs start the calculation of the control method during a while loop of 20 seconds, as described in the previous section, and in the meantime, the RT host executes the fitness function, as depicted in red on Figure 6, which is defined as:

$$
J=\int_{t_{0}}^{t_{\text {end }}}\left|V_{\text {ref }}-V_{\text {sim }}\right| d t
$$

where $V_{\text {ref }}$ is the reference voltage, and $V_{\text {sim }}$ is the simulated voltage. The integral is calculated between $t_{0}$, the starting point, and $t_{\text {end }}$, the simulation time for the single particle. The integral of the absolute error (IAE) penalizes heavily the small error, because smaller but sustained oscillations have a stronger effect if considered with their absolute value, and tends to privilege responses with less oscillation in steady state [17]. This can be considered preferable for this application, where sustained oscillations after the transient must be more penalized.

When the effect of all the particles of a single population has been calculated, the algorithm evaluates these elements:

- Jbest: The best fitness value obtained with a specific population at the $\mathrm{j}$-th iteration.

- $\quad$ xbest: The particle of the $j$-th iteration that has determined the best fitness value.

- Jbest*: The best fitness function among all the fitness functions achieved so far.

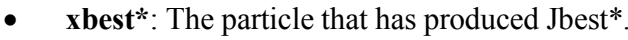

With these values, the update of the velocity of the particles can be calculated, according to this formula (2):

$$
\begin{aligned}
\vec{v}_{i}(j)= & \vec{v}_{i}(j-1)+\varphi_{1} \cdot \operatorname{rand}_{1} \cdot\left(\vec{x}_{\text {best }}-\vec{x}_{i}(j-1)\right) \\
& +\varphi_{2} \cdot \operatorname{rand}_{2} \cdot\left({\overrightarrow{x^{*}}}_{\text {best }}-\vec{x}_{i}(j-1)\right)
\end{aligned}
$$

where $\varphi_{1} \cdot \operatorname{rand}_{1}$ and $\varphi_{2} \cdot \operatorname{rand}_{2}$ are two random weights [8]. The new particles are then calculated composing the old vector of particles and the new velocity vector, as described in (3) [8]:

$$
\vec{x}_{i}(j)=\vec{x}_{i}(j-1)+\vec{v}_{i}(j)
$$

The PSO continues until the number of the updates reaches the stopping criterion, which can be the maximum number of PSO iterations or the maximum number of iteration without having any change on Jbest*.

\section{Simulation Results}

This section demonstrates the usability of the on-line tuning of the control coefficients in a medium-size MVDC shipboard power system and the results obtained after the optimization process in term of fitness function and performance. This approach avoids the use of particular design specifications for the determination of the control parameters. It indeed considers the fitness function the only criterion for the design of the controllers, allowing the control strategies to be compared. According to the literature, every control strategy has many parameters that can be optimized, which can be summarized as follows:

- LSF: The parameters $\omega_{o}$ and $\xi$, starting from the analytical values defined in [9]. The proportional and integral coefficients of the PI controllers.

\begin{tabular}{|c|c|c|c|}
\hline$\omega_{o}$ & $\xi$ & $K_{p}$ & $K_{i}$ \\
\hline $700-300$ & $0.8-0.4$ & $0.5-0.01$ & $100-10$ \\
\hline
\end{tabular}

- Centralized Synergetic: The parameters of the manifold $\Psi_{1}$ of the first converter [6][12].

\begin{tabular}{|c|c|c|}
\hline$a_{11}$ & $a_{12}$ & $a_{13}$ \\
\hline $3500-500$ & $3500-500$ & $100-20$ \\
\hline
\end{tabular}

- LQG: The parameters of the matrix $Q$ and $R$, which vary the optimal gain in the LQG control [12].

\begin{tabular}{|c|c|}
\hline$q$ & $r$ \\
\hline $10-0.01$ & $10-0.01$ \\
\hline
\end{tabular}


- Backstepping: The parameters $c_{1}$ and $c_{2}$ of the Lyapunov function [14].

\begin{tabular}{|c|c|}
\hline$c_{1}$ & $c_{2}$ \\
\hline $25 \mathrm{e}+3-7.5 \mathrm{e}+3$ & $25 \mathrm{e}+3-7.5 \mathrm{e}+3$ \\
\hline
\end{tabular}

- $\quad$ Synergetic: The parameters of the manifold $\Psi$ [12].

\begin{tabular}{|c|c|c|}
\hline$b_{1}$ & $b_{2}$ & $b_{3}$ \\
\hline $3500-500$ & $3500-500$ & $100-20$ \\
\hline
\end{tabular}

The choice of the parameters to optimize and their boundaries comes from the previous experiences on the application of these controllers in the Hardware in the Loop simulations [6] [17].

The results of the application of the PSO algorithm to the control parameters are represented in Figure 7.

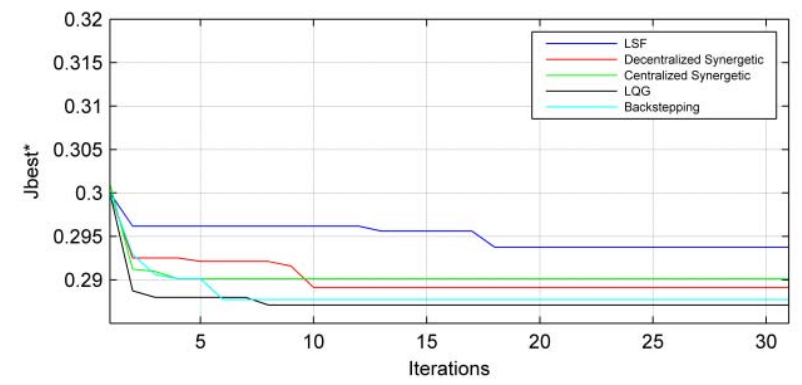

Figure 7. The evolution of Jbest*

By observing the results of the optimization process, the effectiveness of the on-line tuning of the control strategies can be assert. All the controllers have reached a certain minimum level of the fitness function, introducing then a common criterion for the comparison. All the controllers show a comparable value of Jbest*, even though the LSF reveals a value of the fitness function slightly higher. The performance of the voltage stabilizing effect for each control strategy and the effect of the optimization process are depicted from Figure 8 to Figure 12. Every figure depicts the average value of the voltage after a load step of $25 \mathrm{MW}$, starting from a base load of 28 MW. The non-optimal parameters for each controller have been selected among the first generation of particles and do not represent the worst case. The value of the fitness function equal to 0.3 has been chosen as a reference for the selection of the non-optimal particle.

The non-optimal behavior is represented in blue, whereas the red waveform shows the voltage after the application of the PSO. The figures show that the optimization process reduces the oscillations of the voltage for every control strategy. In the LSF, the Synergetic, the LQG and the Decentralized Synergetic the PSO reduced the first overshoot. The use of the integral of the absolute error helps the comparison of the controllers, since the design is based on this unique criterion. The comparison can be considered fair because the IAE mainly penalizes the steady state oscillations and does not privilege any controller during the transient, where the action of the primary controller is predominant.

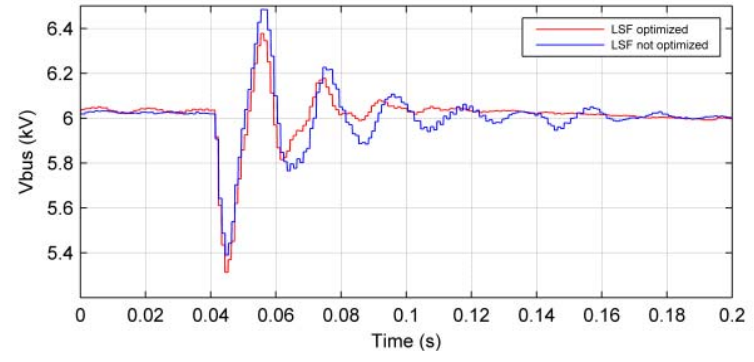

Figure 8. Vbus - LSF

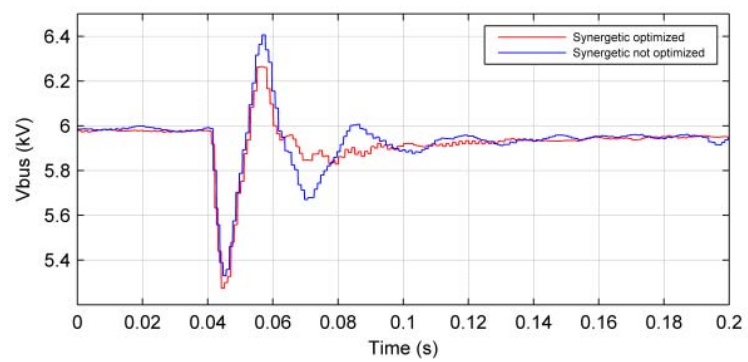

Figure 9. Vbus - Synergetic

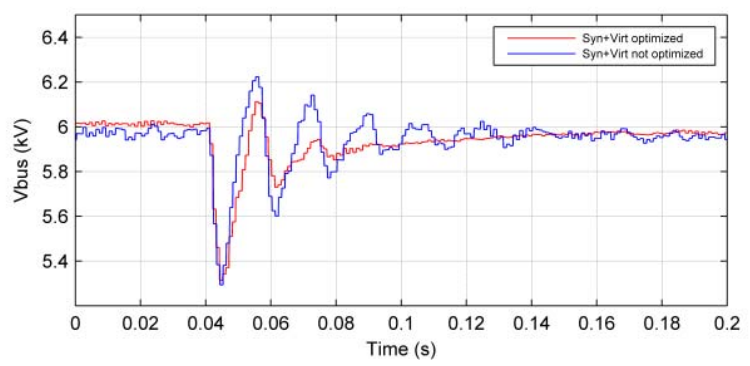

Figure 10. Vbus - Synergetic + Virtual Disturbance

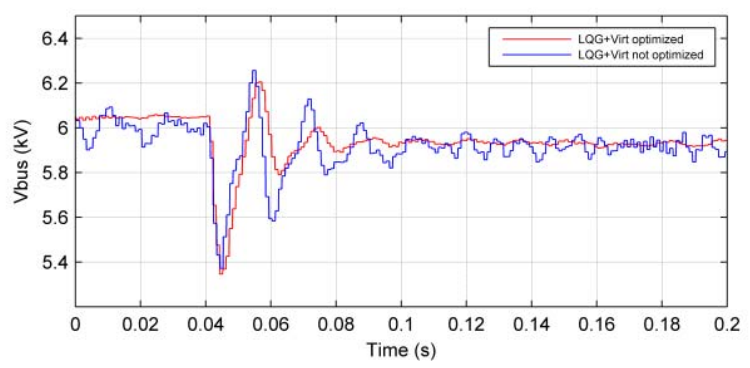

Figure 11. Vbus - LQG + Virtual Disturbance

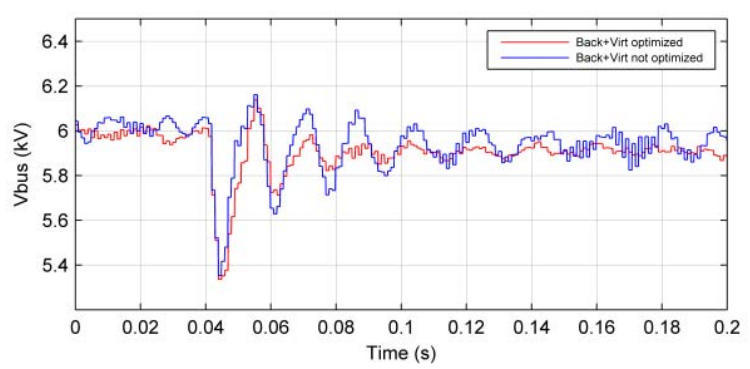

Figure 12. Vbus - Backstepping + Virtual Disturbance 


\section{CONCLUSIONS}

This paper introduced a LabView-based Particle Swarm Optimization (PSO) implementation that has been developed for the online tuning of the control coefficients in the MVDC Shipboard Power System. This optimization process aims at facilitating the control design process for fair performance comparison of different controllers, since the design specification of each control strategy is based on the same fitness function, and for the investigation of the stability of MVDC networks, because it allows to quickly set-up and run a simulation. The PSO calculates combinations of parameters for each control strategy, which drive to a minimum value of the fitness function. This result allows us to state that all the control strategies are able to guarantee a fast and comparable stabilizing action for the MVDC microgrid under test, no matter what controller is chosen, The on-line implementation of the PSO could help the control designer to deal with advanced controllers, which present some difficulties on correlating the control parameters and their effect on the control actions. This can be helpful especially when it is applied to experimental simulation, where the discretized controllers are employed with measured signals. The figures demonstrate the impact of the PSO on optimizing the control strategies and making them comparable.

The PSO can be also able to manage a possible deficiency in the knowledge of the system, which is determined by the complexity of the microgrid itself. Since the design of the controller and the choice of its parameters depend on the knowledge of the microgrid model, the optimization procedure can help compensating the loss of performance of the controller caused by the mismatch between the nominal values of the components and the actual ones, which can vary according to the tolerance range of the component. This capability can be helpful in particular for tuning the centralized controllers, where the equivalent model of the system is required for the evaluation of the control method. A possible future application of the PSO on-line implementation would be testing its ability of coping with a lacking of knowledge of the system, which in many cases reduces the performance of the control action itself.

\section{ACKNOWLEDGMENT}

We gratefully acknowledge the financial support for this project by BMBF (German Federal Ministry of Education and Research) under promotional reference 03EK3566B.

\section{REFERENCES}

[1] Doerry, N., "Naval Power Systems: Integrated power systems for the continuity of the electrical power supply.," IEEE Electrification Magazine, vol.3, no.2, pp.12,21, June 2015

[2] IEEE Recommended Practice for $1 \mathrm{kV}$ to $35 \mathrm{kV}$ Medium-Voltage DC Power Systems on Ships," IEEE Std. 1709-2010 , pp.1,54, Nov. 22010

[3] Emadi, A.; Khaligh, A.; Rivetta, C.H.; Williamson, G.A., "Constant power loads and negative impedance instability in automotive systems: definition, modeling, stability, and control of power electronic converters and motor drives," in Vehicular Technology, IEEE Transactions on , vol.55, no.4, pp.1112-1125, July 2006

[4] Cupelli, M.; Zhu, L.; Monti, A., "Why Ideal Constant Power Loads Are Not the Worst Case Condition From a Control Standpoint," IEEE Transactions on Smart Grid, vol. 6, no. 6, pp. 2596-2606, Nov. 2015.
[5] Sudhoff, S. D.; Glover, S. F.; Zak, S. H.; Pekarek, S. D.; Zivi, E. J.; Delisle, D. E.; Clayton, D.; "Stability analysis methodologies for DC power distribution systems", 13th Int. Ship Control Systems Symposium (SCSS), Orlando, Florida, April 2003

[6] Cupelli, M., "Advanced Control Methods for Robust Stability of MVDC Systems," PhD Dissertation, RWTH Aachen University, 2015

[7] M. Cupelli et al., "Power Flow Control and Network Stability in an AllElectric Ship," in Proceedings of the IEEE, vol. 103, no. 12, pp. 23552380, Dec. 2015.

[8] Y. del Valle, G. K. Venayagamoorthy, S. Mohagheghi, J. C. Hernandez and R. G. Harley, "Particle Swarm Optimization: Basic Concepts, Variants and Applications in Power Systems," in IEEE Transactions on Evolutionary Computation, vol. 12, no. 2, pp. 171-195, April 2008.

[9] Sulligoi, G.; Bosich, D.; Giadrossi, G.; Zhu, L.; Cupelli, M.; Monti, A., "Multiconverter Medium Voltage DC Power Systems on Ships: Constant-Power Loads Instability Solution Using Linearization via State Feedback Control," IEEE Transactions on Smart Grid, vol.5, no.5, pp. 2543,2552 , Sept. 2014

[10] C. Tse, Complex Behavior of Switching Power Converters, CRC Press, London, 2000

[11] Zhu, L.; Liu, J.; Cupelli, M.; Monti, A., "Decentralized Linear Quadratic Gaussian control of multi-generator MVDC shipboard power system with Constant Power Loads," 2013 IEEE Electric Ship Technologies Symposium (ESTS), , pp.308,313, 22-24 April 2013

[12] Cupelli, M.; DeDin, E.; Sulligoi, G.; Monti, A., "Case Study of Voltage Control for MVDC Microgrids with Constant Power Loads Comparison between Centralized and Decentralized Control Strategies," to appear in 2016 18th IEEE Mediterranean Electrotechnical Conference (MELECON),

[13] Cupelli, M.; Moghimi, M.; Riccobono, A.; Monti, A., "A comparison between synergetic control and feedback linearization for stabilizing MVDC microgrids with constant power load," 2014 IEEE PES Innovative Smart Grid Technologies Conference Europe (ISGT-Europe), pp.1,6, 12-15 Oct. 2014

[14] Cupelli, M.; Mirz, M., M.; Monti, A., "Application of Backstepping to MVDC Ship Power Systems with Constant Power Loads," Electrical Systems for Aircraft, Railway and Ship Propulsion (ESARS), 2015, pp.1,5, 3-5 Mar. 2015

[15] Cupelli, M.; Mirz, M., M.; Monti, A., “A Comparison of Backstepping and LQG control for stabilizing MVDC Microgrids with Constant Power Loads," 2015 IEEE Eindhoven PowerTech (POWERTECH), pp.1,6, 29 June -2 July 2015

[16] Guerrero, J.M.; Vasquez, J.C.; Matas, J.; de Vicuña, L.G.; Castilla, M., "Hierarchical Control of Droop-Controlled AC and DC Microgrids-A General Approach Toward Standardization," in Industrial Electronics, IEEE Transactions on, vol.58, no.1, pp.158-172, Jan. 2011

[17] Y. L. Abdel-Magid and M. A. Abido, "AGC tuning of interconnected reheat thermal systems with particle swarm optimization," Electronics, Circuits and Systems, 2003. ICECS 2003, pp. 376-379 Vol.1

[18] Cupelli, M.; de Paz Carro, M.; Monti, A., "Hardware in the Loop Implementation of Linearizing State Feedback on MVDC Ship Systems and the Significance of Longitudinal Parameters," Electrical Systems for Aircraft, Railway and Ship Propulsion (ESARS), 2015 , pp.1,5, 3-5 Mar. 2015

[19] Cupelli, M.; de Paz Carro, M.; Monti, A., "Hardware in the Loop Implementation of a Disturbance Based Control in MVDC Grids," 2015 IEEE Power and Energy Society General Meeting (PES), pp.1,5, 26-30 July 2015 